\title{
Was leisten Wissenschaftsverlage heute eigentlich noch?
}

Lorenz M. Hilty

Der Lektor am Telefon war ausgesprochen hilfsbereit. Er hatte sich über den Inhalt des Lehrbuches, das ich im Auftrag eines Professors überarbeitete, ausführlich Gedanken gemacht und gab mir in stundenlangen Telefongesprächen seine Erfahrungen zu Stil und Layout weiter. So lernte ich im Laufe eines Jahres einiges über die professionelle Produktion von Fachbüchern. Später sah ich den Band mit einigem Stolz in Buchhandlungen ausliegen, übrigens zu einem für Studierende erschwinglichen Preis.

Das war Anfang der 1990er-Jahre. Die Verlage hatten den Schriftsatz schon an die Autoren und ihre PCs ausgelagert, beschäftigten aber noch Lektoren, die mitdachten und sich Zeit für ein Buchprojekt nahmen, um die Qualität des Ergebnisses sicherzustellen. Diese positiven Erfahrungen hatte ich damals mit Springer, aber auch mit anderen renommierten Verlagen gemacht, in denen ich sowohl Monografien als auch Sammelbände publiziert habe.

Nichts ahnend fasste ich deshalb, mehr als 20 Jahre später, den Entschluss, erneut bei Springer zu veröffentlichen. Einen Sammelband, der die besten Beiträge einer internationalen Konferenz enthalten sollte. Wir wollten keine Proceedings im üblichen Sinne herausgeben, sondern einen sorgfältig editierten Band mit Kapiteln, die aufeinander Bezug nehmen. Weil Autoren heute an bibliometrischen Indikatoren gemessen werden, war es ein Glücksfall, dass Springer eine passende Reihe mit dem Hinweis „Now indexed by ISI Proceedings, EICompendex, DBLP, SCOPUS, Google Scholar and Springerlink" bewarb. Mein Team organisierte einen Peer-Review-Prozess für die Buchkapitel, um die Qualität des Inhalts nach heute üblichen Standards zu sichern. Einem erfolgreichen Buch schien nichts mehr im Weg zu stehen. Doch es sollte ganz anders kommen - ein Drama in fünf Akten.

Erster Akt. Auf meine Anfrage hin zeigt Springer Interesse, unser Buch in der besagten Reihe $\mathrm{zu}$ veröffentlichen und schätzt den voraussichtlichen Verkaufspreis für die Printversion auf „99,99 Euro“. Das klingt zwar nicht gerade günstig, liegt aber im Rahmen des heute Üblichen für ein Werk dieses Umfangs. Wir unterzeichnen einen Vertrag, die Autoren beginnen ihre Arbeit, wir versorgen sie mit den - laut Springers Website - für diese Reihe maßgeblichen „Author Guidelines“ und einem vielversprechenden Paket von „Author Tools“. Einige Vorgaben sind sehr ungewöhnlich, beispielsweise das Format für Überschriften der dritten und vierten Gliederungsebene. Aus der Erfahrung heraus, dass Autoren solche Regeln gern ignorieren und dann unzählige Varianten produzieren, die nachträglich nur mit riesigem Aufwand zu vereinheitlichen sind, weisen wir unsere 47 Autorinnen und Autoren zusätzlich auf solche Besonderheiten hin. Auch darauf, dass alle Abbildungen für die Printversion auch ohne Farbe verständlich sein müssen.

Zweiter Akt. Zahlreiche Rückfragen zeigen uns, dass es Unklarheiten in den Guidelines gibt und Inkonsistenzen mit den Author Tools. Einige Autoren haben außerdem andere Guidelines von der Springer-Website heruntergeladen und sind nun

DOI 10.1007/s00287-015-0896-7

(C) Springer-Verlag Berlin Heidelberg 2015

Lorenz M. Hilty

Institut für Informatik, Universität Zürich,

Zürich, Schweiz

E-Mail: hilty@ifi.uzh.ch 


\section{Zusammenfassung}

Einen Sammelband in einer renommierten Buchreihe herauszugeben, ist heute eine Erfahrung der besonderen Art. Noch konsequenter als bei Zeitschriften werden alle Arbeiten mit Ausnahme des Marketings auf Herausgeber und niedrig qualifizierte Arbeitskräfte in Billiglohnländern abgewälzt. Erfahrungsbericht eines Herausgebers.

überzeugt, dass diese für unsere Reihe gelten. Wir senden Listen mit detaillierten Fragen an den Verlag. Diese werden zwar beantwortet, die Antworten werfen aber häufig neue Fragen auf. Eine Lektorin schickt uns relativ spät eine neue Version von Richtlinien, die wir noch nie gesehen haben. Und den überraschenden Hinweis, dass wir uns „um Formatierungen nicht zu kümmern" brauchen, weil das Buch ,professionell gesetzt“" werde. Bitte prägen Sie sich diesen Satz gut ein, um das Kommende daran zu messen.

Dritter Akt. Eigentlich ist es eine gute Nachricht, dass sich Profis um das Layout kümmern werden und wir als Herausgeber damit nichts zu tun haben. Aber abgesehen davon, dass die Nachricht etwas spät kommt und im Widerspruch zu den Instruktionen im Web steht, fehlt mir der Glaube an dieses Wunder. Ich versuche weiterhin, ein möglichst einheitliches und ausgereiftes Manuskript abzuliefern. Wir schärfen den Autoren weiterhin ein, ab der dritten Gliederungsebene nicht zu nummiereren (wie in den Richtlinien verlangt) und lösen viele andere Detailprobleme. Wir achten darauf, dass die Literaturlisten aller Kapitel einheitlich formatiert sind. Und natürlich bestehen wir weiterhin darauf, dass alle Abbildungen auch ohne Farbe verständlich sein müssen, weil die Printversion bei diesem Preis ohne Farbbilder auskommen muss. All dies verursacht erheblichen Aufwand. Wir, die Herausgeber, betrachten es als Investition, die in einem weiteren Schritt - der Endkorrektur - allen Beteiligten Aufwand sparen und die Einhaltung des ambitionierten Zeitplans ermöglichen wird. Dann liefern wir die rund 470 Seiten an Springer ab.

Vierter Akt. Per E-Mail meldet sich ein freundlicher Herr von einer Firma in Chennai, Indien. Er sei im Auftrag von Springer für die Produktion unseres Buches zuständig, ,,in accordance with the Sprin- ger style guidelines“. Wir sind gespannt, welche Guidelines das nun sein werden. Als wir die PDFDatei des gesamten Buches zur Korrektur erhalten, ist die Antwort klar: Keine. Die Korrekturfahnen sehen aus, als hätte man sie mit einem Zufallsgenerator editiert. Für Überschriften ab der dritten Gliederungsebene gibt es neuerdings neun verschiedene Varianten, manchmal sogar mehrere in einem einzigen Kapitel. Zudem wurden in einigen Kapiteln die Überschriften gar nicht als solche erkannt. Keine der neun Varianten ist übrigens konform mit den Springer-Richtlinien für diese Reihe, einige davon enthalten sogar nummerierte Abschnitte. Sie erinnern sich: Genau das haben wir den Autoren mühsam ausgeredet.

Doch das ist lange nicht das einzige Problem: Platzhalter, die wir eingesetzt haben, um auf andere Kapitel des gleichen Bandes zu verweisen, wurden nicht aufgelöst, obwohl das mit mehren Personen bei Springer per E-Mail vereinbart war; in den Literaturlisten aller Kapitel wurden die Titel sämtlicher Quellen automatisch auf Kleinschreibung umformatiert, einschließlich Abkürzungen, sodass wir in Hunderten von Fällen beispielsweise „,it“ in „IT“, „un“ in „UN“, ,oecd“ in „OECD“ zurückkorrigieren müssen; in einigen Kapiteln wurden Dutzende von Referenzen aus dem Text entfernt und dann „Author Queries“ generiert wie diese: „References $[56,57$, $58,59,60,61,62,63,64,65,66,67,68,69]$ are given in list but not cited in text. " Solche Schäden lassen sich nur mit Rückgriff auf das ursprüngliche Manuskript und auch nur mit einem Verständnis für den Inhalt (jawohl, das gibt es noch: Inhalt) in mühsamer Handarbeit reparieren.

Die Liste der Zerstörungen ließe sich fortsetzen. Ich sende eine seitenlange detaillierte Beschwerde an den indischen Auftragnehmer und zur Kenntnis an Springer. Sie enthält den Satz: „It is inevitable that someone who tries to understand will now look at both the current text and the author manuscripts and decide what has to be done. "Sie ahnen schon: Diesen „someone“ wird es nicht geben, außer wir machen die Arbeit selbst. Wir sitzen in der Falle, weil wir das Buch auf einer kurz bevorstehenden Konferenz präsentieren wollen. Wer weiß, welche Verzögerungen mit einem Verlagswechsel und möglichen juristischen Auseinandersetzungen verbunden wären. Lassen wir das Projekt scheitern, ist alle schon investierte Arbeit - besonders die der 47 Autorinnen und Autoren - entwertet. 
Problematisch ist, dass wir den Text in dieser Phase des Prozesses nicht mehr selbst ändern können und jede einzelne Korrektur explizit annotieren müssen. Viele Korrekturen führen in der Umsetzung durch die Profis zu neuen Fehlern, die von uns gefunden und in der nächsten Runde wieder korrigiert werden müssen. Das erinnert mich an alte Zeiten - an Softwareentwicklung im Batch-Betrieb. Es ist ein Gefühl, wie jemandem per Telefon das Töpfern beizubringen. Auf unsere Beschwerden reagieren sie mit langatmigen E-Mails, in denen sie um Nachsicht betteln: „Please bear with us!“ Also üben wir uns in Nachsicht, d. h. wir machen ihre Arbeit, machen Nächte und Wochenenden durch zur Lösung lächerlicher Probleme, die vollkommen vermeidbar gewesen wären. Nachsicht durch Nachtschicht.

Springer hält es nicht für nötig, sich in unsere Kommunikation mit den Indern einzuschalten. Ein bestechendes Geschäftsmodell: Lass unqualifizierte Arbeiter zusammen mit gutmütigen Wissenschaftlern etwas erarbeiten, wobei Letztere kostenlos die Qualitätssicherung übernehmen, auch dann noch, wenn das Projekt zum Fass ohne Boden wird. Und lass dir alle Rechte am Ergebnis abtreten - als Gegenleistung dafür, dass du diesen Prozess mal eben schlecht und recht organisierst.

Fünfter Akt. Das Buch ist auf dem Markt. Die Geburtswehen, die es verursacht hat, sind ihm nicht anzusehen. Springer verkauft es nun zum stolzen Preis von 203 Euro, 253 Schweizerfranken oder 259 US-Dollar pro Exemplar (Printversion, Softcover). Der Unterschied zum ursprünglich genannten Preis ist nur teilweise dadurch zu erklären, dass es nun 474 statt 300 Seiten umfasst. Außerdem liege dem ursprünglich genannten Preis ein Irrtum seitens Springer zugrunde, wie man uns sagt. Einzelne Kapitel kosten online übrigens um die 25 Euro, was hochgerechnet etwa dem dreieinhalbfachen Preis der Printversion entspricht.

Doch der Preis bleibt nicht die einzige Überraschung. 26 der insgesamt 119 Abbildungen sind in Farbe gedruckt worden. Allerdings nicht diejenigen, bei denen die Farbe am meisten zum Verständnis beigetragen hätte. Niemand hat uns über diese Entscheidung informiert, niemand hat über die Bedeutung der Farben in den Abbildungen nachgedacht. Autoren melden sich bei uns und beklagen sich: „Wenn ich gewusst hätte, dass Farbdruck möglich ist ..."
Es ist offensichtlich, dass es beim Verlag keine Person gab, die sich inhaltlich über dieses Buch Gedanken gemacht hätte. Ich vermute außerdem, dass niemand einen Überblick über den gesamten Prozess hatte. Wir hatten mit insgesamt sieben Personen bei Springer und seinem indischen Auftragnehmer zu tun. Diese wussten voneinander meist nicht, was mit uns abgesprochen war, wenn sie sich auch redlich bemüht haben, uns aus ihrer jeweils begrenzten Perspektive heraus zu unterstützen.

Eine weitere Überraschung war das Erscheinungsjahr: Obwohl am 22. August 2014 erschienen (auch als Printversion), steht im Impressum das Jahr 2015. Das muss uns nicht weiter stören, da das Buch dadurch nominell länger frisch bleibt, aber die Seriosität dieser Praxis ist zumindest diskutierbar.

Sieben Monate nach seinem Erscheinen ist unser Buch übrigens immer noch nicht ,indexed by ISI Proceedings, EI-Compendex, DBLP, SCOPUS, Google Scholar and Springerlink“, wie es auf der Website von Springer so schön hieß, und entscheidend sind hier ISI und SCOPUS. Die Autoren beginnen sich bei mir zu beschweren, weil die Publikation für bibliometrische Indikatoren nicht zählt, solange Springer sein Versprechen nicht einlöst. Meine wiederholten Nachfragen bewirken nichts - außer dass Springer den Hinweis auf der Website subtil ändert. Es heißt neuerdings: „Indexing: The books of this series are submitted to ISI Proceedings, EI-Compendex, DBLP, SCOPUS, Google Scholar and Springerlink“. Die Formulierung ,submitted to “ ist näher an der Wahrheit als die alte („now indexed by"), da Springer nach eigenen Angaben gar keinen direkten Einfluss auf die meisten dieser Dienste hat, da sie ja von Konkurrenten betrieben werden, und die Aufnahme eines Werks nur vorschlagen kann. Das bisherige Versprechen an die Autoren war also zumindest fahrlässig. Die Autoren scheinen sich damit scharenweise ködern zu lassen. In der besagten Reihe sind seit 2013 über 180 Titel erschienen, im Mittel also mehr als ein Band pro Woche. Eine Fließbandproduktion ohne jegliche Qualitätssicherung durch den Verlag. Springer gibt insgesamt rund 4500 Buchreihen heraus. Das ist leicht möglich, wenn man damit so gut wie nichts mehr zu tun hat - außer Werbung zu machen, natürlich.

Bis vor zwei, drei Jahrzehnten haben Verlage noch Themen identifiziert, Autoren gefunden, beraten und betreut. Ihre Reputation haben sie erworben, indem sie die inhaltliche und technische 
Qualität der Werke sicherstellten. Außerdem sind sie erhebliche finanzielle Risiken eingegangen (und mussten deshalb wählerisch sein), da die Auflagen nicht beliebig klein sein konnten. Heute dagegen, im Zeitalter von „Print on Demand“, ist das finanzielle Risiko nahe null. Die Produktion wird, wie wir gesehen haben, in Billiglohnländer ausgelagert und die Qualitätssicherung komplett auf die Herausgeber und Autoren abgewälzt. Warum ist ein Wissenschaftsverlag lukrativer als eine Notenpresse? Richtig, die Banknoten müsste man selber drucken.

Mit diesem kritischen Bericht möchte ich nicht etwa die Mitarbeitenden des indischen Auftragnehmers für ihre mangelnde Ausbildung oder Erfahrung kritisieren. Was ich kritisiere, ist die rücksichtslose Externalisierung von Kosten durch den SpringerVerlag, der billige Arbeitskräfte einsetzen kann, weil er gleichzeitig die Qualitätssicherung an weitgehend aus Steuergeldern bezahlte Herausgeberinnen und Herausgeber abwälzt. Dadurch gehen die Kosten nach unten - die Verkaufspreise werden gleichzeitig erhöht, weil die Universitäten über ihre Bibliotheken und Konferenzen die von ihnen finanzierten Produkte bereitwillig zurückkaufen. Die Verlagstätigkeit reduziert sich auf Werbung mit einer Marke, die ihren Ruf der Arbeit einer früheren Generation von Profis - wirklichen Profis - zu verdanken hat.

Angesichts dieser Realitäten drängt sich mir die Frage auf, ob sich denn niemand um den Ruf einer erfolgreichen Marke sorgt - offenbar nicht. Eine naheliegende Erklärung ist, dass die renommier- ten Wissenschaftsverlage schon lange und in voller Absicht vom Glanz vergangener Zeiten leben. Noch umgibt die großen Marken ein Mythos von Qualität und Glaubwürdigkeit. Die Zukunft wird zeigen, wie lange man daraus noch Profit schlagen kann, ohne sich um die Einlösung des damit verbundenen Anspruchs zu bemühen - wie lange es dauert, bis der Gaul zu Tode geritten ist. Nachhaltig ist das nicht.

Diesen Text habe ich den Betroffenen vor der Veröffentlichung zur Kenntnis gegeben. Springer hat daraufhin seine Anstrengungen verstärkt, den Band wie angekündigt bei SCOPUS erfassen zu lassen. Dies ist geschehen, exakt acht Monate nach Erscheinen des Buches. Die Erfassung durch ISI steht weiterhin aus.

Vermutlich ist das hier geschilderte Problem nicht spezifisch für den Springer-Verlag. Kolleginnen und Kollegen berichten von ähnlichen Erfahrungen mit anderen Giganten der Branche. Es spricht für die Trennung von redaktionellen und kommerziellen Belangen bei Springer, dass dieser Artikel im Informatik-Spektrum erscheinen konnte.

Lorenz M. Hilty ist Professor am Institut für Informatik der Universität Zürich und leitet die gemeinsame Forschungsgruppe „Informatik und Nachhaltigkeit" der Universität Zürich und der Eidgenössischen Materialprüfungs- und Forschungsanstalt Empa. Er ist außerdem Delegierter für Nachhaltigkeit der Universität Zürich und Affiliated Professor am „Center for Sustainable Communications“, CESC, des Royal Institute of Technology, KTH, Stockholm. 